\title{
A kinetic-Monte Carlo perspective on active matter
}

\author{
Juliane U. Klamser, ${ }^{1, a)}$ Sebastian C. Kapfer, 2, b) and Werner Krauth ${ }^{1,},{ }^{(2)}$ \\ 1) Laboratoire de Physique Statistique, Département de physique de l'ENS, Ecole Normale Supérieure, PSL Research University, \\ Université Paris Diderot, Sorbonne Paris Cité, Sorbonne Universités, UPMC Univ. Paris 06, CNRS, 75005 Paris, \\ France. \\ ${ }^{2)}$ Theoretische Physik 1, FAU Erlangen-Nürnberg, Staudtstr. 7, 91058 Erlangen, Germany
}

(Dated: 18 December 2018)

We study non-equilibrium phases for interacting two-dimensional self-propelled particles with isotropic pair-wise interactions using a persistent kinetic Monte Carlo (MC) approach. We establish the quantitative phase diagram, including the motility-induced phase separation (MIPS) that is a commonly observed collective phenomena in active matter. In addition, we demonstrate for several different potential forms the presence of two-step melting, with an intermediate hexatic phase, in regions far from equilibrium. Increased activity can melt a two-dimensional solid and the melting lines remain disjoint from MIPS. We establish this phase diagram for a range of the inter-particle potential stiffnesses, and identify the MIPS phase even in the hard-disk limit. We establish that the full description of the phase behavior requires three independent control parameters.

\section{INTRODUCTION}

Active matter is an important field of research that considers particle systems whose microscopic components are characterized by systematic persistent dynamic rules and by various types of mutual interactions. On a microscopic scale, the persistent dynamics breaks the detailed-balance condition (underlying all of equilibrium physics) and defines active matter as out-of-equilibrium systems.

Many different models of active matter have been proposed. They feature a wide range of self-propelled dynamics and of mutual interactions. A great many theoretical studies employ either Langevin or molecular dynamics ${ }^{1-6}$ in order to model persistent motion. Recently, a kinetic Monte Carlo (MC) approach was proposed ${ }^{7 / 8}$ as a minimal model for active matter in two dimensions.

Although the primary interest in theoretical models of active matter comes from the non-equilibrium nature, their properties can often be connected to their equilibrium counterparts that are realized in the zero-persistence limit. This limit is of particular interest in two dimensions, where equilibrium particle systems with short-range interactions cannot crystallize ${ }^{9}$. Nevertheless, it was established that, from the high-temperature (low-density) liquid regime towards the low-temperature (high-density) solid regime, twodimensional equilibrium particle systems normally undergo two phase transitions 10 . 12 . These transitions describe the passage into and out of a hexatic phase that is sandwiched between the liquid and the solid phase (see Table II). In this work, we are concerned with two-dimensional models with repulsive inverse-power-law interactions, for which the twostep melting scenario in equilibrium is firmly established ${ }^{13} 14$.

In this work, we extend our earlier findings for a special case ${ }^{8}$ and show that kinetic MC generically reproduces motility-induced phase separation (MIPS) in two-dimensional

\footnotetext{
a)Electronic mail: Juliane_klamser@yahoo.de.

b) Electronic mail: Sebastian.kapfer@fau.de.

${ }^{\text {c) Electronic mail: Werner.krauth@lps.ens.fr. }}$
}

TABLE I. Decay of correlation functions in the liquid, hexatic, and solid phases in two-dimensional particle systems.

\begin{tabular}{llcr}
\hline \hline Order & Liquid phase & Hexatic phase & Solid phase \\
\hline Positional & short-range & $\begin{array}{c}\text { short-range } \\
\text { Orientational }\end{array}$ & $\begin{array}{r}\text { quasi-long-range } \\
\text { short-range }\end{array}$ \\
long-range & quasi-long-range \\
\hline \hline $\mathrm{a} \propto \exp (-r / \xi)$ & & \\
$\mathrm{b} \propto r^{-\alpha}$ & & \\
$\mathrm{c} \propto$ constant &
\end{tabular}

active-particle systems with a wide range of inverse-powerlaw interactions including the hard-sphere limit. We also confirm the stability of the hexatic phase up to considerable values of the activity, and conjecture that it is indeed stable at any value of the activity. We finally confirm that MIPS is generically a liquid-gas transition under the kinetic MC dynamics. Moreover, it is decoupled from the melting transitions. This separation can be understood in the limit of infinitesimal MC steps from the scaling behavior of the MIPS phase transition and the melting transitions.

The work is organized in the following order. In Section II the essential elements of the kinetic MC algorithm are described, the interplay of persistence with interactions is illustrated on a simple case of two particles in one dimension (1D), and possible anisotropy effects in two dimensions (2D) are analyzed. In Section III, we discuss the effect of the stiffness of the interparticle potential on the full quantitative phase diagram of two-dimensional particle systems on the activitydensity plane. The continuous-time limit of the kinetic MC dynamics is discussed in Section IV] with a focus on the number of relevant parameters.

\section{MODEL: KINETIC MC}

The kinetic MC algorithm with which we model active dynamics consists of the standard Metropolis filter combined with a memory term for the proposed moves. The memory term is characterized by a time scale $\tau$, which allows for a smooth tuning from a passive motion (described by equilib- 

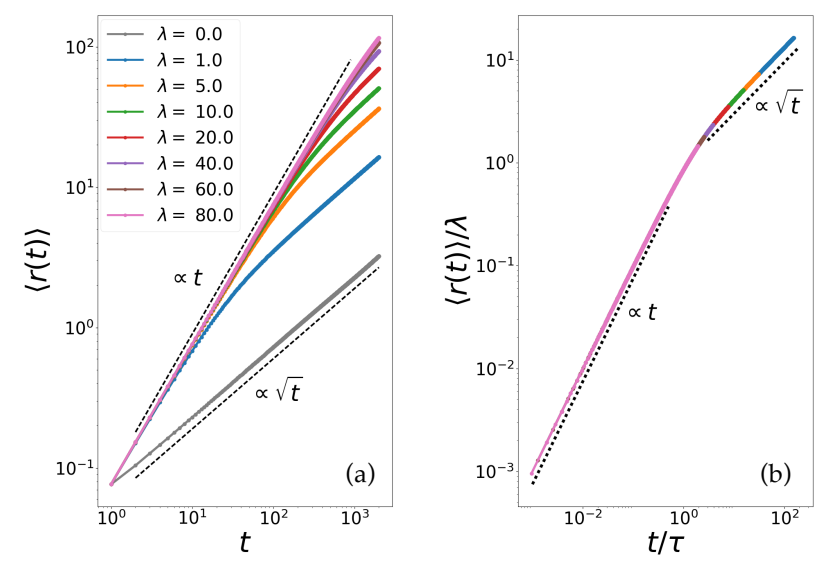

FIG. 1. Mean absolute displacement $v s$. time $t$ for a single particle. (a) The crossover from ballistic to diffusive motion shifts to larger $t$ with increasing activity, measured in terms of the persistence length $\lambda$ (see eq. (1)). (b) Data collapse illustrating the crossover from $\propto t$ to $\propto \sqrt{t}$ at around $(1,1)$ expressed using eqs $(1)$ and (2).

rium statistical mechanics) to a self-propelled/persistent particle motion, where a single particle moves ballistically (mean square displacement $\propto t^{2}$ ) for times $t \ll \tau$ and moves diffusively (mean square displacement $\propto t$ ) for times $t \gg \tau$ (see Fig. 11(a)).

In contrast to active Brownian particles ${ }^{15}$, the velocity amplitude fluctuates in the MC dynamics. The dynamics is comparable with the active Ornstein-Uhlenbeck process (see e.g. Ref. 16), where the velocity performs a random walk in a harmonic potential. Similarly, in the discrete-time kinetic $\mathrm{MC}$ approach the increment performs a random walk in a box with reflecting boundary conditions. For a single particle in two dimensions, the $x$ - and $y$-components of the increment $\left(\varepsilon_{x}(t), \varepsilon_{y}(t)\right)$ at time $t$ are sampled from two independent Gaussian distributions of standard deviation $\sigma$ and the mean corresponds to the previously sampled increment $\left(\varepsilon_{x}(t-1)\right.$ or $\varepsilon_{y}(t-1)$, respectively). This walk is confined in $x$ and $y$ by reflecting boundaries at $x= \pm \delta$ and $y= \pm \delta$. In two dimensions the average distance a single particle covers before changing the direction is given by ${ }^{8}$ the persistence length

$$
\lambda \simeq 0.62 \frac{\delta^{3}}{\sigma^{2}}
$$

and the persistence time is given by

$$
\tau=\frac{8}{\pi^{2}} \frac{\delta^{2}}{\sigma^{2}}
$$

This characteristic length and time scales are confirmed in numerical simulations. For example, the crossover from ballistic to diffusive motion in Fig. 11(b) appears in the rescaled time-dependence of the mean absolute displacement around the point $(t / \tau=1,\langle r(t)\rangle / \lambda=1)$.

In the many-particle case, the increment for each particle performs its proper random $\epsilon$-walk, independent of the other particles. Interactions between particles are introduced by the Metropolis filter. In one kinetic MC step, a particle $i$ is chosen

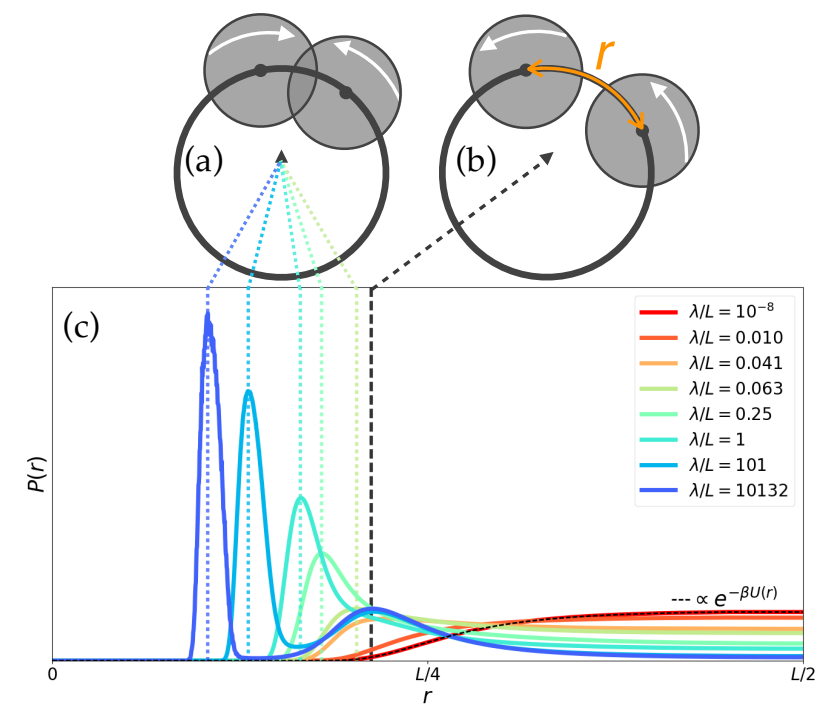

FIG. 2. Persistence for two 1D particles on a ring (line of length $L$ with periodic boundary conditions). (a) and (b) indicate the two states of the system that lead to the local maxima of the paircorrelation function. White arrows indicate the sign of the displacements $\varepsilon_{i}$. (c) Pair correlation function $P(r)$ for different $\lambda$. Maximum jump length $\delta=L / 40 \lambda$ is varied by changing $\sigma$. Data for $n=6$, $u_{0} \beta=1$. The unit length scale is set by $\gamma$ in eq. (4).

at random. The change of its position $\left(\boldsymbol{r}_{i}(t+1)=\boldsymbol{r}_{i}(t)+\right.$ $\left.\boldsymbol{\epsilon}_{i}(t)\right)$ is accepted with probability

$$
P\left(E^{\prime} \rightarrow E\right)=\min \left[1, e^{-\beta \Delta E)}\right]
$$

where $\Delta E=E-E^{\prime}$ is the total energy change caused by the particle displacement $\boldsymbol{r}_{i}(t) \rightarrow \boldsymbol{r}_{i}(t+1)$. The parameter $1 / \beta=$ $k_{\mathrm{B}} T$ is now an energy scale rather than a temperature. The random $\boldsymbol{\epsilon}$-walk persists whether the resulting displacement is accepted or not. For a detailed description of the kinetic MC approach, see Ref. 8 .

\section{A. 1D Model for Persistence}

Kinetic MC, via the memory present in its displacements, generates persistence in a manner that differs from equilibrium systems. This has far-ranging consequences for manyparticle systems, but the effects of a memory term in the equations of motion can already be studied for $N=1$ or $N=2$ particles. Here we study the case of two particles on a ring (a line of length $L$ with periodic boundary conditions), interacting with an inverse-power-law pair potential that we will use throughout this work

$$
U(r)=u_{0}\left(\frac{\gamma}{r}\right)^{n}
$$

where $\gamma$ reduces to the particle diameter in the hard-disk limit $n \rightarrow \infty$. The 1D inter-particle distance $r$ in eq. (4) is easily generalized to higher dimensions. 
Fig. 2 shows the probability distribution $P(r)$ of the interparticle distance. The maximum jump length $\delta$ is kept constant and the persistence length $\lambda$ is varied by changing $\sigma$. As the interaction is repulsive, the two particles repel each other for small $\lambda$, and the Boltzmann weight is maximal at $r=L / 2$ for $\lambda=0$ (see Fig. 22(c)). For increased $\lambda$, a peak appears at small $r$, and its position decreases with increasing $\lambda$. This means that particles are more probable to be near each other, which is due to the case, where the particles try to move against each other (see Fig. 2(a)). The higher the persistence length, the stronger the force the particles push against the interparticle potential barrier and therefore, the peak position shifts. This shows that the self-propulsion force increases with $\lambda$ or equivalently with the persistence time $\tau$, and the shift is a result of the larger number of attempts in the Metropolis filter to increase the total energy.

At finite $\lambda$, the original (Boltzmann) peak at $r=L / 2$ shifts to smaller $r$ and takes on a position that is independent of $\lambda$. This peak appears due to arrangements where one particle "hunts" the other circling around the ring with $\left|\varepsilon_{\text {hunter }}\right|>$ $\left|\varepsilon_{\text {hunted }}\right|$ (see Fig. 2(b)). For this to have a non-negligible probability, the role of the slower and faster particle has to be stable for a sufficient time span, which explains that the peak appears only after overcoming a certain threshold in $\lambda$. The independence of the peak position on $\lambda$ is understood from the following argument. Moves of the slower particle are always accepted by the Metropolis filter, as they decrease the total energy. In contrast, moves of the faster particle are often rejected as they increase the total energy. This leads to a competition where the slower particle increases $r$ in every attempt, and the faster particle tries to decreases $r$, but does not succeed in every attempt, thereby leading to an average "hunting distance" $r$, which is independent of $\lambda$ (see Fig. 2(c)).

The bimodal probability distribution of $P(r)$ (see Fig. 2) is a consequence of the non-constant velocity amplitude and thus in contrast to e.g. active Brownian particles. The result clearly shows that the self-propulsion force in the kinetic MC dynamics depends on the persistence time $\tau$. This is not the case in the Langevin approaches of active Brownian particles or the active Ornstein-Uhlenbeck process. However, as discussed in Section IV] the persistent length $\lambda$ is the relevant measure for activity is this dynamics.

\section{B. Anisotropic effects}

In our kinetic MC algorithm, displacements $\varepsilon=\left(\varepsilon_{x}, \varepsilon_{y}\right) \in$ $[-\delta, \delta]^{2}$ are confined to a square box rather than being sampled from an isotropic distribution (as for example a circle of radius $\delta$ ). (The 2D Gaussian distribution of the OrnsteinUhlenbeck process is also isotropic.) Although the square box is chosen for simplicity only, it is useful to check that it does not induce anisotropies. This trivially follows in the passive limit for the steady-state probability distribution because of the detailed-balance condition.

At small times $(t \ll \tau)$, the reflecting boundary conditions for the sampling scheme of the displacements introduces some degree of anisotropy in the two-dimensional single-
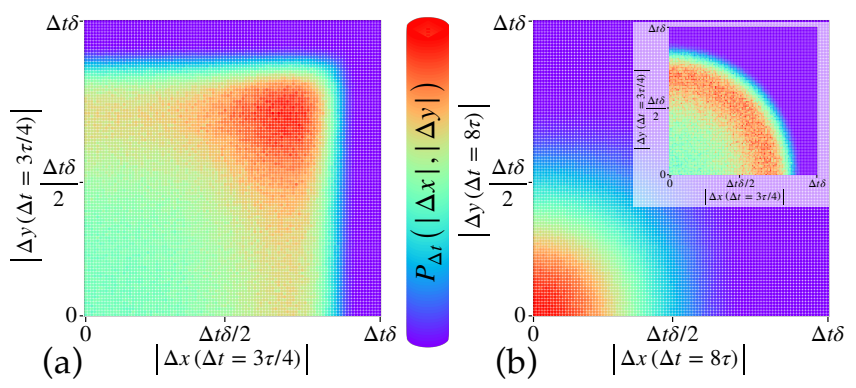

FIG. 3. Kinetic-MC displacement distribution $P_{\Delta t}(|\Delta x|,|\Delta y|)$, in time $\Delta t$, for a single particle (infinite system). (a): Anisotropic total displacement for small times $\left(\Delta t=\frac{3}{4} \tau\right)$. (b): Isotropic total displacement for large times $(\Delta t=8 \tau)$. Inset in (b): Displacement distribution for a circular reflecting boundary for small times $\left(\Delta t=\frac{3}{4} \tau\right)$. (Linear color code with zero at purple.)
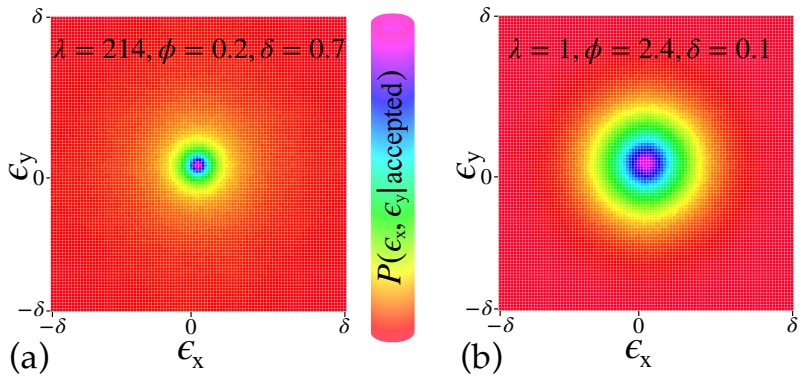

FIG. 4. Two-dimensional probability distribution of the accepted displacements $P\left(\varepsilon_{x}, \varepsilon_{y} \mid\right.$ accepted $)$. (a) Many-body system in the MIPS region. (b) In the solid near the solid-hexatic transition at a density far above the equilibrium melting lines $(n=6, N=10976)$.

particle dynamics (see Fig. 3(a)). The probability distribution $P_{\Delta t}(|\Delta x|,|\Delta y|)$ of the absolute particle displacement $\Delta x$ (or $\Delta y$ ) in the $x$ (or $y$ ) component in a time $\Delta t$ is anisotropic for $\Delta t<\tau$ (see Fig. 3.a)) whereas, without the square box, this displacement (which is constructed from identically distributed, independent Gaussians in both dimensions) would be isotropic. However, the isotropy is reinstalled for $t \gg \tau$ (see Fig. 3. (b)). As $\tau=0$ in the passive limit, the particle dynamics is isotropic for all times 17 . The anisotropy for $t<\tau$ could also be avoided by choosing a circular sampling box of radius $\delta$ with reflective boundary conditions for the displacements (see Fig. 3(b)). However, such a choice would be more costly to implement.

We conjecture that the square box in fact renders anisotropic the long-time dynamics neither for $N=1$ nor for the many-body case. In the dilute case (where $\lambda$ is much smaller than the mean free path) the kinetic MC dynamics effectively reverts to the detailed-balance dynamics as interactions between particles happen at the diffusive time scale. At higher densities, anisotropy in the many-body properties might arise if the probability distribution of the accepted displacements is itself anisotropic. However, this is not the case (see Fig. 47. At higher densities, all large proposed displacements have a vanishing probability to be accepted by the Metropolis filter, thus leading to an effectively isotropic dynamics. Therefore, the Metropolis filter effectively realizes 


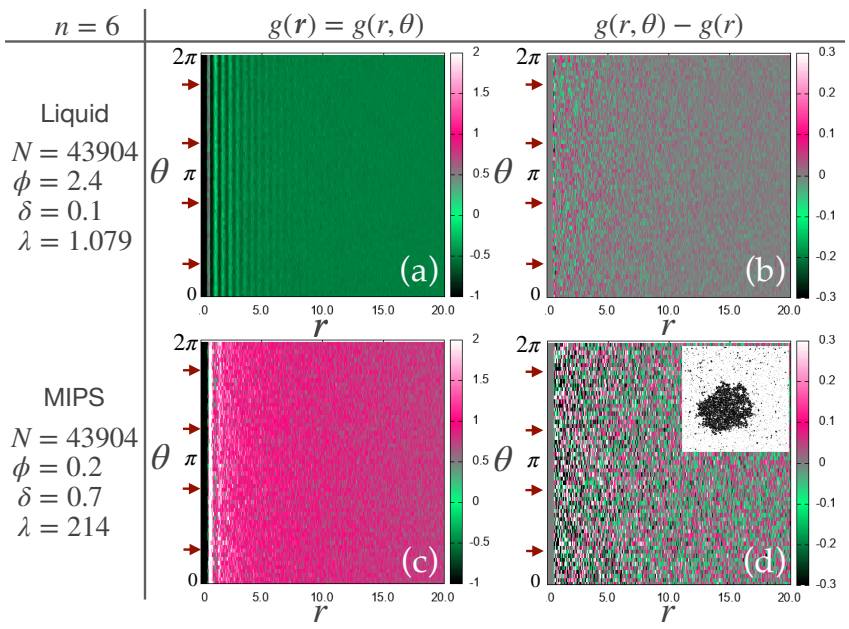

FIG. 5. Effective isotropic dynamics $(n=6, N=43904)$. (a) and (c): pair-correlation function $g(r, \theta)$ in polar coordinates averaged over 100 configurations. (b) and (d): difference between $g(r, \theta)$ and its angular average $g(r)$. The red arrows indicate $\pi / 4,3 \pi / 4,5 \pi / 4$ and $7 \pi / 4$. Inset in (d): snapshot of configuration showing MIPS corresponding to (c) and (d).

a circular reflecting $\varepsilon$-sampling box without additional computational cost. Pair-correlation functions are also found to be perfectly isotropic at high density, both in the motilityinduced liquid phase (at a density deep inside the equilibrium solid phase) and in the MIPS region (see Fig. 5).

\section{TWO-DIMENSIONAL SIMULATION RESULTS}

Our simulations are performed in an ensemble of $N$ particles confined to a rectangular ${ }^{8}$ box of volume $V$ with periodic boundary conditions. The density $\phi=\gamma^{2} N / V$ (with $\gamma$ from eq. (4)) is varied by changing $V$. In the simulations $\delta$ is kept constant and the activity is varied by changing $\sigma$.

\section{A. Full phase diagram and the effect of stiffness}

Fig. 6(a) shows the full phase diagram for the potential in eq. (4) with $n=16$ on the $\phi-\lambda$ plane. At all $\lambda$, the equilibrium two-step melting transition 14 is recovered. For increasing $\lambda$, the melting lines shift to higher densities. Remarkably, the hexatic phase separating the liquid and solid phases is stable even far from equilibrium. This non-equilibrium two-step melting can be induced either by reducing the density (just as in equilibrium) or by increasing the persistence length. In addition to these melting transitions, at low $\phi$ but high $\lambda$, a motility-induced liquid-gas coexistence region opens up. It is separated form the melting transitions by a disordered fluid phase. This generalizes the phase diagram under the same dynamics, but for $n=6$, found previously $\sqrt{8}$. A change of $n$ (see eq. (4)) only shifts the positions of the phase boundaries (see Fig. 6(b)). As for the equilibrium melting transitions 14 , both the liquid-hexatic and the hexatic-solid phase bound-
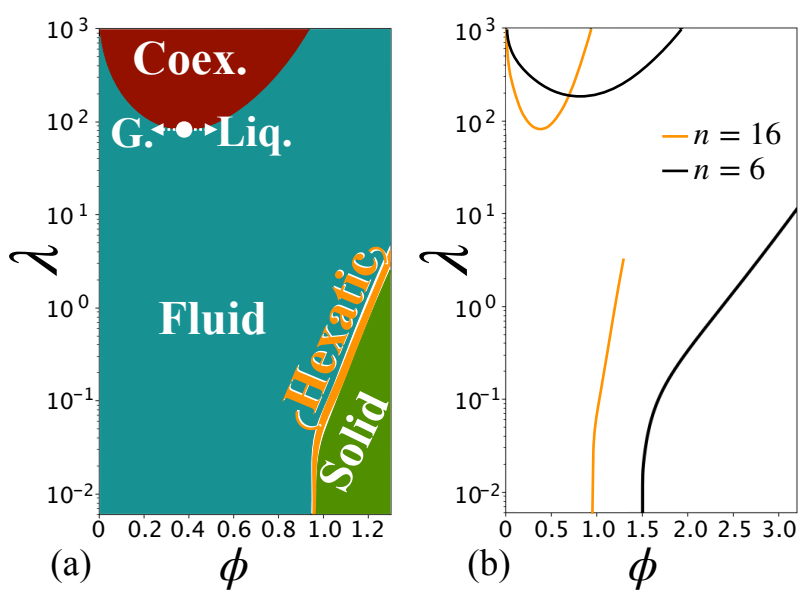

FIG. 6. Phase diagram as a function of density $\phi$ and persistence length $\lambda$ (a): Soft-disk potential with $n=16$. (b): Comparison of phase boundaries 8 for $n=6$ with the steeper $n=16$ case $(\delta=0.1$, $\left.\gamma=1, u_{0} \beta=1\right)$. MIPS is always separated from the melting transitions.

aries shift at constant persistence length $\lambda$ to smaller densities with increasing $n$.

Increasing $\lambda$ shifts the melting transitions to higher densities. However, the shift is smaller for larger $n$, resulting in steeper transition lines (see Fig. 6(b)). At the same time, the onset of the motility-induced liquid-gas coexistence shifts to smaller $\phi$ and $\lambda$ and the coexisting region shrinks. This ensures that the liquid-gas coexistence and the melting transitions remain disjoint. In Section III C, we argue that there is no singular change in the phase diagram even in the hard-disk limit $n \rightarrow \infty$.

\section{B. Non-equilibrium two-step melting}

In equilibrium, the Mermin-Wagner theorem forbids longrange translational (i.e. crystalline) order in a $2 \mathrm{D}$ particle system with short-range interactions 1819 . However, at large densities, particles can arrange in locally hexagonal configurations. This can lead to two different high-density phases 10,12 , which are characterized by different degrees of orientational and positional order (see Table II). In this section, we define these measures of order and use them to quantify the two-step melting far from equilibrium.

The local bond-orientational order parameter $\psi_{6}\left(\boldsymbol{r}_{i}\right)$ measures the six-fold orientation near a particle $i$. It is defined as

$$
\psi_{6}\left(\boldsymbol{r}_{i}\right)=\frac{1}{\text { number of neighbors } j \text { of } i} \sum_{j} \exp \left(6 \iota \theta_{i j}\right),
$$

where $\iota$ is the imaginary unit and $\theta_{i j}$ it the angle enclosed by the $x$-axis and the connection line between particle $i$ and its neighbor $j$. Here, we use the Voronoi construction to identify neighbors and $\psi_{6}\left(\mathbf{r}_{i}\right)$ is calculated with Voronoi weights 20 . 
Then, the correlation function

$$
g_{6}(r) \propto\left\langle\sum_{i, j}^{N} \psi_{6}^{\star}\left(\boldsymbol{r}_{i}\right) \psi_{6}\left(\boldsymbol{r}_{j}\right) \delta\left(r-r_{i j}\right)\right\rangle
$$

is a measure of the correlation of the local six-fold orientational order at distance $r$ and its decay is used to quantify the degree of orientational order in the system (see Table I).

The direction-dependent pair-correlation function $g(x, y)$ provides a measure for the positional order. This twodimensional histogram is averaged over different configurations $C$ after re-aligning $\sqrt{13} g_{C}(x, y)$ such that the $\Delta \mathrm{x}$-axis points in the direction of the global orientation parameter $\Psi_{6}(C)=$ $\sum_{i}^{N} \psi_{6}\left(\boldsymbol{r}_{i}\right)$ of $C$. Then, the decay of, e.g. $g(x, 0)$ determines the degree of positional order.

The correlation functions $\left(g(x, 0)\right.$ and $\left.g_{6}(r)\right)$, allow one to identify the two-dimensional phases by the properties summarized in Table I. In equilibrium, the Kosterlitz-ThoulessHalperin-Nelson-Young theory provides an additional selection criterion ${ }^{10}-12$, where the exponent (defined in Table I) $\alpha \leq 1 / 4$ for the orientational order and $\alpha \leq 1 / 3$ for the positional order give theoretical bounds for the hexatic phase. However, these bounds are not shown to apply outside equilibrium. We thus identify the phases by the characteristic decay of $g(x, 0)$ and $g_{6}(r)$.

Orientational and positional correlation functions change as the system melts from solid to liquid passing through the hexatic phase (see Fig. 7, at a density far above the equilibrium melting point). Our simulations clearly identify solid state points with power-law decay in $g(x, 0)$ and constant $g_{6}(r)$, hexatic state points with exponential decay of $g(x, 0)$ and quasi-long-range order in $g_{6}(r)$, and liquid state points, where both correlations decay exponentially. Snapshots illustrate these different phases (see Fig. 7 and Table I). The power-law exponent in $g_{6}(r)$ grows when approaching the liquid-hexatic transition and therefore, weakening the order in the hexatic phase. This behavior of $g_{6}(r)$ is in agreement with equilibrium studies ${ }^{14}$ and with our earlier results 8 obtained with the same dynamics for $n=6$.

We check that our simulations indeed reach the steady state (as in earlier work ${ }^{8}$ ) by verifying the convergence of the spatial correlation functions to the same steady state starting from a crystalline and a liquid initial particle arrangement. These very time-consuming computations assure that the defining decays of the correlation functions obtained in the hexatic phase reflect the physical system and not a bias introduced by the initial condition.

\section{Motility-induced phase separation}

At sufficiently low densities and high activities, a liquidgas coexistence region opens up with a roughly U-shaped phase boundary (see Fig.6.a)). Following an analysis of local densities, which was applied earlier ${ }^{8}$ for $n=6$, we confirm that also for $n=16$ the densities ( $\phi_{\text {Liquid }}$ and $\left.\phi_{\text {Gas }}\right)$ of the two coexisting phases depend on $\lambda$ but not on the global density $\phi$. Therefore, the low-density boundary of the MIPS region
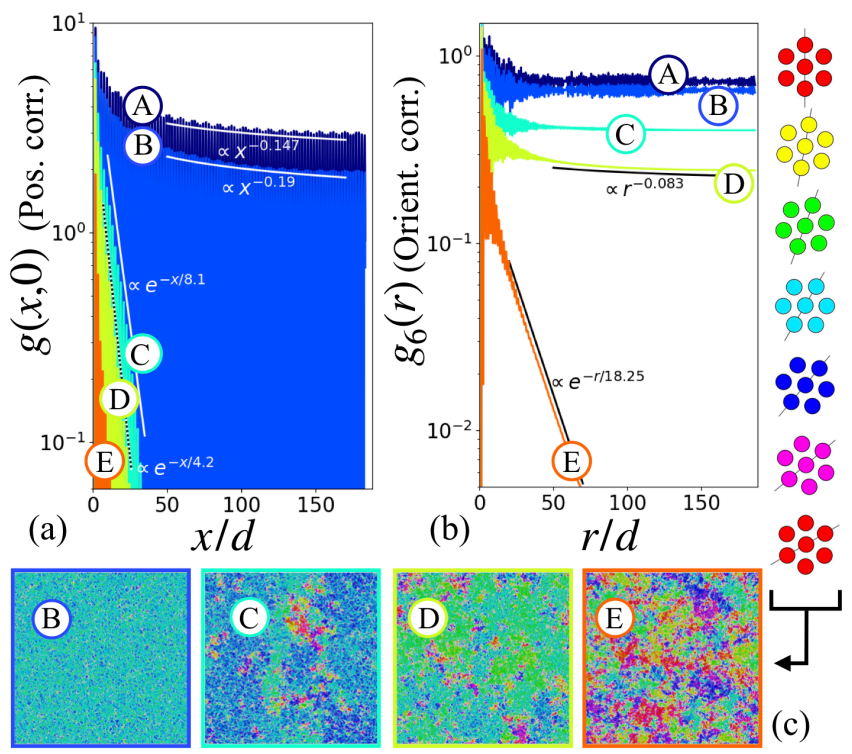

FIG. 7. Two-step melting for $n=16$ at high density. (a): Positional correlation $g(x, y=0)(b)$ orientational correlation $g_{6}(r)$. Particles in (c) are color-coded according to local orientational order $\psi_{6}$. "A" and "B" are solid (algebraic $g(x, 0)$, long-range $g_{6}(r)$ ). "C" and "D" are hexatic (exponential $g(x, 0)$, algebraic $g_{6}(r)$ ). "E" is liquid (both decays exponential $).\left(d=(\pi N / V)^{-1 / 2}, \phi=1.2, N=43904, \delta=\right.$ $0.1, u_{0} \beta=1$, "A": $\lambda=0.5$, "B": $\lambda=0.7$, "C": $\lambda=1.0$, "D": $\lambda=1.1$, "E": $\lambda=1.4)$

in Fig. 6(a) is given by ${ }^{8} \phi=\phi_{\text {Gas }}(\lambda)$ and the high-density boundary by $\phi=\phi_{\text {Liquid }}(\lambda)$, respectively.

A much discussed question ${ }^{2 / 36 / 21 / 22}$ concerns the nature of the two phases at coexistence. We can clearly identify the high-density phase as liquid, and MIPS as a liquid-gas coexistence. The particles in the snapshots in Fig. 8 are $\psi_{6}$-colorcoded (see Fig. 7 for definition), illustrating short-ranged orientational order in the liquid phase. We do not observe that the orientational correlation in the liquid phase increases with increasing $\lambda$. Even at very high activities (e.g. at $\lambda=4 \times 10^{3}$ in Fig. $8(a)$ ), the local orientational order changes upon length scales of the order of the interparticle distance (also compare with Fig.7(c) point E).

We do not observe any quantitative difference with the orientational order in MIPS previously observed ${ }^{8}$ for $n=6$ for the same kinetic MC dynamics. Furthermore, we also recover MIPS in the hard-disk system in the form of a liquid-gas coexistence (see Fig. 8(b)). We conjecture from these findings that the separation of the MIPS region from the melting transitions is a generic feature of self-propelled particles, at least within kinetic MC dynamics.

\section{RELEVANT PARAMETERS}

So far (as our previous work ${ }^{8}$ ), we have considered the phase diagram as a function of the persistence length and the density, keeping the maximum step size $\delta$ constant (see Fig. 6). However, $\delta$ has a profound influence on the phase 


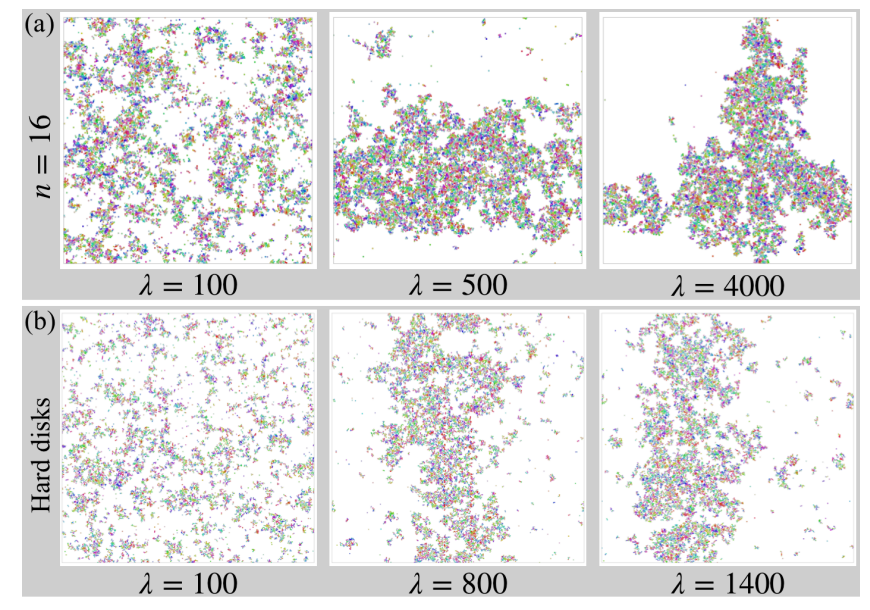

FIG. 8. Short-range order in MIPS for $n=16$ and for hard disks. (a) Snapshots for $n=16$ at $\phi=0.4$. (b): Snapshots for hard disks $(n \rightarrow$ $\infty)$ at $\phi=0.2$. The liquid phase is clearly identified by a short-ranged orientational correlation illustrated by the $\psi_{6}$ color code defined in Fig.7 Data for $N=10976, \delta=0.1, \beta u_{0}=1$.

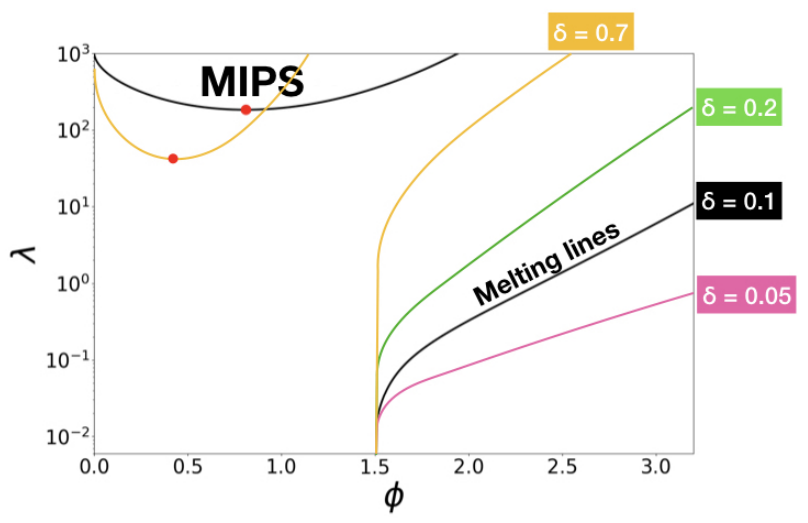

FIG. 9. Phase boundaries (MIPS and melting transitions) for different step sizes $\delta$. Data for $n=6, u_{0} \beta=1.0$.

boundaries (see Fig. 9). Keeping $\phi$ constant, the melting lines shift to smaller activities as $\delta$ is decreased, while in contrast the MIPS phase boundary shifts to larger activities. In this section, we study this $\delta$-dependence of melting and of MIPS.

The kinetic MC dynamics depends on three parameters $(\delta$, $\lambda, \phi$ ). We now show that MIPS (seen at high $\lambda$ ) and the melting close to equilibrium are described by a different reduced set of relevant parameters in the $\delta \rightarrow 0$ limit. The single relevant parametel ${ }^{14}$, which describes the melting transitions for inverse-power-law potentials is not commensurate with the reduced parameters for MIPS, as it does not capture the critical melting density in the passive limit. Therefore, there are separate descriptions of MIPS and the melting transitions.

Here we introduce the Master equation as a stochastic descriptions of our kinetic MC dynamics, in addition to a Langevin description and the associated Fokker-Planck equation. We also compare the dimensional reduction of the relevant parameters with other stochastic models of active matter.

\section{A. A simple argument}

We first address the question of relevant parameters with a simple argument for a single particle. (A more detailed analysis is presented in the following Section IVB and Section IVC) We consider the small- $\delta$ limit, which is justified for the choice of parameters used in the simulations. For a single particle in a $1 \mathrm{D}$ confining potential $U(x)$, the kinetic $\mathrm{MC}$ rule is approximated by the following discrete-time $(k=0,1,2, \ldots)$ dynamics:

$$
\begin{aligned}
& \varepsilon_{k+1}=\varepsilon_{k}+r_{k}+R\left(\frac{\varepsilon_{k}}{\delta}\right), \\
& x_{k+1}=x_{k}+\varepsilon_{k} f\left(x_{k}, \varepsilon_{k}\right),
\end{aligned}
$$

where $r_{k}$ is a Gaussian random number with $\left\langle r_{k}\right\rangle=0$, $\left\langle r_{k} r_{k^{\prime}}\right\rangle=\sigma^{2} \delta_{k, k^{\prime}}$ and

$$
f(x, \varepsilon)=\min \left\{1, \exp \left(-\frac{U(x+\varepsilon)-U(x)}{k_{\mathrm{B}} T}\right)\right\}
$$

is the acceptance rate of the Metropolis filter in eq. (3). The reflecting boundary at $\varepsilon= \pm \delta$ is denoted by $R$ (without specifying it rigorously) and $\delta_{k, k^{\prime}}$ is the Kronecker delta.

Defining a set of rescaled coordinates,

$$
t=k \delta, \quad v(t)=\frac{\varepsilon_{k}}{\delta}, \quad \xi(t)=\frac{r_{k}}{\delta^{2}}, \quad x(t)=x_{k},
$$

and taking the small- $\delta$ limit, we get

$$
\begin{aligned}
\dot{v}(t) & =\xi(t)+R(v(t))+\mathscr{O}(\boldsymbol{\delta}) \\
\dot{x}(t) & =v(t) f(x(t), v(t) \boldsymbol{\delta})+\mathscr{O}(\boldsymbol{\delta})
\end{aligned}
$$

where $\langle\xi(t)\rangle=0,\left\langle\xi(t) \xi\left(t^{\prime}\right)\right\rangle=\lambda^{-1} \delta\left(t-t^{\prime}\right)+\mathscr{O}(\delta / \lambda)$, with $23=\delta^{3} / \sigma^{2}$ and we use a continuous limit of the Kronecker delta $\delta_{k, k^{\prime}} \simeq \delta \delta\left(t-t^{\prime}\right)$, with $\delta(x)$ being the Dirac delta function. Moreover, in the small- $\delta$ limit, using Taylor expansion, we get

$$
\frac{U(x+v \delta)-U(x)}{k_{\mathrm{B}} T}=\Gamma_{1} v U^{\prime}(x)\left[1+\frac{\delta v}{2} \frac{U^{\prime \prime}(x)}{U^{\prime}(x)}+\cdots\right],
$$

where $\Gamma_{1}=\delta / k_{\mathrm{B}} T$. As long as $\delta U^{\prime \prime}(x) / U^{\prime}(x) \ll 1$, from eq. (7), we get $f[x(t), v(t) \delta] \simeq h[x(t), v(t)]$, with

$$
h[x(t), v(t)]=\min \left\{1, \exp \left(-\Gamma_{1} v U^{\prime}(x)\right)\right\} .
$$

This gives a continuous dynamics for $\delta \rightarrow 0$

$$
\begin{aligned}
& \dot{v}(t)=\xi(t)+R(v(t))+\mathscr{O}(\boldsymbol{\delta}), \\
& \dot{x}(t)=v(t) h(x(t), v(t))+\mathscr{O}(\boldsymbol{\delta})+\mathscr{O}\left(\delta \frac{U^{\prime \prime}(x)}{U^{\prime}(x)}\right) .
\end{aligned}
$$

Clearly, this rescaled dynamics depends on only two relevant parameters, namely

$$
\Gamma_{1}=\frac{\delta}{k_{\mathrm{B}} T}, \text { and } \lambda=\frac{\delta^{3}}{\sigma^{2}} .
$$

However, for this description to be valid, the subleading terms have to be negligible, leading to the following range of validity for the scaling in eq. 13): 
a) $\delta \ll \lambda\left(\right.$ from $\left.\left\langle\xi(t) \xi\left(t^{\prime}\right)\right\rangle\right)$,

b) $\delta \ll \lambda^{-1 / 2}$ (from eq. 12a) ), and

c) $\delta \ll 1$ and $\delta \ll U^{\prime}(x) / U^{\prime \prime}(x)$ (from eq. (12b)).

Therefore, the scaling expressed in the two-parameter reduction in eq. (13) breaks down in a) the passive limit, b) at very high persistence lengths (for constant $\phi$ ), and c) at high density (for constant $\lambda$ ).

\section{B. Stochastic description of the MC dynamics}

We begin the rigorous analysis by considering a single particle in a $1 \mathrm{D}$ confining potential $U(x)$. The kinetic MC dynamics is Markovian in the $(x, \varepsilon)$ space. The discrete kinetic MC time is denoted by $k=0,1,2, \ldots$. The conditional probability for a transition $\left(y, \mathcal{\varepsilon}^{\prime}\right) \rightarrow(x, \boldsymbol{\varepsilon})$ in one time step is given by the Markov matrix

$$
M\left(x, \varepsilon \mid y, \varepsilon^{\prime}\right)=g\left(\varepsilon, \varepsilon^{\prime}\right) W_{\varepsilon}(x, y),
$$

where, $\varepsilon$ is sampled with probability $g\left(\varepsilon, \varepsilon^{\prime}\right)$ and $W_{\varepsilon}(x, y)$ is due to the Metropolis filter. It can be shown ${ }^{24}$ that

$$
\begin{aligned}
g\left(\varepsilon, \varepsilon^{\prime}\right)= & \frac{1}{2 \delta}+\frac{1}{\delta} \sum_{m=1}^{\infty} \exp \left[-\frac{\pi^{2} \sigma^{2} m^{2}}{8 \delta^{2}}\right] \\
& \cos \left(\frac{m \pi}{2 \delta}(\varepsilon+\delta)\right) \cos \left(\frac{m \pi}{2 \delta}\left(\varepsilon^{\prime}+\delta\right)\right),
\end{aligned}
$$

whereas the Metropolis filter in eq. (3) yields

$$
W_{\varepsilon}(x, y)=f(y, \varepsilon) \delta(x-y-\varepsilon)+[1-f(y, \varepsilon)] \delta(x-y),
$$

with $f(x, \varepsilon)$ as defined in eq. (77). Using this in the corresponding discrete-time Master equation

$$
P_{k+1}(x, \varepsilon)=\int d y \int_{-\delta}^{\delta} d \varepsilon^{\prime} M\left(x, \varepsilon \mid y, \varepsilon^{\prime}\right) P_{k}\left(y, \varepsilon^{\prime}\right)
$$

gives

$$
\begin{array}{r}
P_{k+1}(x, \varepsilon)=\int d \varepsilon^{\prime} g\left(\varepsilon, \varepsilon^{\prime}\right) P_{k}\left(x, \varepsilon^{\prime}\right)+\int d \varepsilon^{\prime} g\left(\varepsilon, \varepsilon^{\prime}\right) \\
\left\{f(x-\varepsilon, \varepsilon) P_{k}\left(x-\varepsilon, \varepsilon^{\prime}\right)-f(x, \varepsilon) P_{k}\left(x, \varepsilon^{\prime}\right)\right\} .
\end{array}
$$

This describes the exact time evolution of the probability $P_{k}(x, \varepsilon)$ in the kinetic MC dynamics 25 . In the passive limit, this satisfies the standard detailed-balance condition with respect to the Boltzmann distribution (see Appendix A).

\section{Rescaled coordinates}

To determine the relevant number of control parameters, we use the scaled coordinates defined in eq. (8) in the Master equation (eq. (17p)):

$$
\begin{aligned}
\tilde{P}_{t+\delta}(x, v)= & \int d v^{\prime} \tilde{g}\left(v, v^{\prime}\right) \tilde{P}_{t}\left(x, v^{\prime}\right) \\
& +\int d v^{\prime} \tilde{g}\left(v, v^{\prime}\right)\left\{f(x-\delta v, \delta v) \tilde{P}_{t}\left(x-\delta v, \delta v^{\prime}\right)\right. \\
& \left.-f(x, \delta v) \tilde{P}_{t}\left(x, \delta v^{\prime}\right)\right\},
\end{aligned}
$$

with $P_{k}(x, \varepsilon)=\tilde{P}_{t}(x, v) / \delta$ and $g\left(\varepsilon, \varepsilon^{\prime}\right)=\tilde{g}\left(v, v^{\prime}\right) / \delta$.

We have shown in Section IV A that the effective number of control parameters can be reduced by taking the small- $\delta$ limit. In this limit $f(x, \delta v) \simeq h(x, v)$ (see eq. (11)). Using a Taylor expansion, this leads to

$$
\begin{aligned}
\frac{\partial}{\partial t} \tilde{P}_{t}(x, v)= & a_{1}(v) \partial_{v} \tilde{P}_{t}(x, v)+\frac{a_{2}(v)}{2} \partial_{v}^{2} \tilde{P}_{t}(x, v) \\
& -\int d v^{\prime} \tilde{g}\left(v, v^{\prime}\right) v \frac{\partial}{\partial x}\left\{h(x, v) \tilde{P}_{t}\left(x, v^{\prime}\right)\right\}+\cdots,
\end{aligned}
$$

where we used $\int d v^{\prime} \tilde{g}\left(v, v^{\prime}\right)=1$ and

$$
a_{m}(v)=\frac{1}{\delta} \int d v^{\prime}\left(v^{\prime}-v\right)^{m} \tilde{g}\left(v, v^{\prime}\right) .
$$

$a_{m}(v)$ can be computed using eq. (15). Alternatively, we can use the free case

$$
\tilde{g}\left(v, v^{\prime}\right)=\frac{1}{\sqrt{2 \pi \delta / \lambda}} \exp \left[-\frac{\left(v-v^{\prime}\right)^{2}}{2 \delta / \lambda}\right]
$$

in combination with a zero-current condition on $\tilde{P}_{t}(x, v)$ for the reflecting boundary. This simplifies the calculation of $a_{m}(v)$, giving $a_{1}(v)=0$, and $a_{2}(v)=1 / \lambda$, which leads to the FokkerPlanck equation at small $\delta$,

$$
\frac{\partial}{\partial t} \tilde{P}_{t}(x, v)=\frac{1}{2 \lambda} \partial_{v}^{2} \tilde{P}_{t}(x, v)-\frac{\partial}{\partial x}\left\{v h(x, v) \tilde{P}_{t}(x, v)\right\}
$$

with the reflecting boundary condition

$$
\frac{\partial}{\partial v} \tilde{P}_{t}(x, v)=0 \quad \text { for } v= \pm 1 .
$$

This Fokker-Planck equation is equivalent to the coupled Langevin equation in eq. (12) of the approximate dynamics. In consistence with the analysis in Section IV A, this FokkerPlanck equation depends on two parameters $\left(\lambda\right.$ and $\left.\Gamma_{1}\right)$ given in eq. (13). However, as noted earlier, this is only true in a certain range of parameters where the subleading terms are negligible. In particular, the Fokker-Planck equation in eq. 20) fails to describe the passive limit $(\lambda=0)$, where the relevant parameter is differen $t^{14}$ from $\Gamma_{1}$.

To describe the passive limit, a diffusive scaling with $\delta$ is required

$$
t=k \delta^{2}, \quad v=\frac{\varepsilon}{\delta}, \quad x=x .
$$

Starting from eq. (17) and following a similar procedure as for the derivation of eq. 20a, leads to the well-known FokkerPlanck equation for a passive particle in a potential

$$
\frac{\partial \tilde{P}_{t}(x)}{\partial t}=\frac{1}{k_{\mathrm{B}} T} \frac{\partial}{\partial x}\left[U^{\prime}(x) \tilde{P}_{t}(x)\right]+\frac{\partial^{2}}{\partial x^{2}}\left[\tilde{P}_{t}(x)\right] .
$$

A detailed derivation ${ }^{24}$ finds back that in the passive limit the inverse-power-law potential is described by a single control parameter, as is well knowr ${ }^{14}$. 
In conclusion, the number of relevant parameters can be reduced when $\delta$ is small: the persistent limit has two relevant parameters $\left(\Gamma_{1}\right.$ and $\left.\lambda\right)$ and the passive limit $(\lambda=0)$ has a single parameter $\Gamma_{0}$ (see eq. (36)). However, $\Gamma_{1}$ does not converge to $\Gamma_{0}$ for $\lambda \rightarrow 0$. Therefore, the order of the limits $\delta \rightarrow 0$ and $\lambda \rightarrow 0$ cannot be exchanged.

\section{Multi-particle case}

The discussion in Section IV B can be generalized to the multi-particle case. The single-particle Fokker-Planck equation in eq. 20a generalizes to the $N$-particle Fokker-Planck equation

$$
\frac{\partial}{\partial t} P_{t}[\mathbf{x}, \mathbf{v}]=\frac{1}{2 \lambda} \sum_{i} \frac{\partial^{2}}{\partial v_{i}^{2}} P_{t}[\mathbf{x}, \mathbf{v}]-\sum_{i} \frac{\partial}{\partial x_{i}}\left\{v_{i} h_{i}\left(\mathbf{x}, v_{i}\right) P_{t}[\mathbf{x}, \mathbf{v}]\right\},
$$

where particles interact via the inter-particle potential $U(\mathbf{x})$, with $\mathbf{x}=\left\{x_{1}, \cdots, x_{N}\right\}$ and

$$
h_{i}\left(\mathbf{x}, v_{i}\right)=\min \left\{1, \exp \left(\Gamma_{1} v_{i} F_{i}[\mathbf{x}]\right)\right\}
$$

with the force on particle $i$

$$
F_{i}[\mathbf{x}]=-\frac{\partial U[\mathbf{x}]}{\partial x_{i}}
$$

The reflecting boundary condition in the velocity space is

$$
\frac{\partial}{\partial v_{i}} P_{t}[\mathbf{x , v}]=0 \quad \text { for } v_{i}= \pm 1
$$

The Fokker-Planck 22] in the passive limit has a very similar multi-particle generalization.

\section{Power-law interaction potential}

The numerical studies presented in this work are for the inverse-power-law potential in eq. (4). The force on particle $i$ is

$$
F_{i}[\mathbf{x}]=n u_{0} \gamma^{n} \sum_{j \neq i} \frac{\operatorname{sgn}\left(x_{i}-x_{j}\right)}{\left|x_{i}-x_{j}\right|^{n+1}}
$$

For this specific choice, two dimensionless parameters characterize the persistent many-particle behavior. These can be obtained from the mean inter-particle distance

$$
d=\frac{L}{N}=\frac{\gamma}{\phi}, \quad \text { for } 1 \mathrm{D}
$$

with $L$ being the system size or, equivalently, from the dimensionless density

$$
\phi=\frac{\gamma N}{L}, \quad \text { for } 1 \mathrm{D} .
$$

Then, using the scaled coordinates

$$
\tilde{t}=\frac{t}{d}, \quad \tilde{v}(\tilde{t})=v(t), \quad \tilde{x}(\tilde{t})=\frac{x(t)}{d},
$$

in eq. 23, we obtain the rescaled Fokker-Planck equation

$$
\frac{\partial}{\partial \tilde{t}} \tilde{P}_{\tilde{t}}[\tilde{\mathbf{x}}, \tilde{\mathbf{v}}]=\frac{1}{2 \tilde{\lambda}} \sum_{i} \frac{\partial^{2}}{\partial \tilde{v}_{i}^{2}} \tilde{P}_{\tilde{t}}[\tilde{\mathbf{x}}, \tilde{\mathbf{v}}]-\sum_{i} \frac{\partial}{\partial \tilde{x}_{i}}\left\{\tilde{v}_{i} \tilde{h}_{i}\left(\tilde{\mathbf{x}}, \tilde{\mathbf{v}}_{i}\right) \tilde{P}_{\hat{t}}[\tilde{\mathbf{x}}, \tilde{\mathbf{v}}]\right\},
$$

with

$$
\tilde{h}_{i}\left(\tilde{\mathbf{x}}, \tilde{v}_{i}\right)=\min \left\{1, \exp \left(\Gamma \tilde{v}_{i} \sum_{j \neq i} \frac{\operatorname{sgn}\left(\tilde{x}_{i}-\tilde{x}_{j}\right)}{\left(\tilde{x}_{i}-\tilde{x}_{j}\right)^{n+1}}\right)\right\},
$$

and

$$
\tilde{\lambda}=\frac{\lambda}{d}, \quad \text { and } \quad \Gamma=\frac{n u_{0} \gamma^{n} \delta}{k_{\mathrm{B}} T d^{n+1}}
$$

These two parameters $(\tilde{\lambda}$ and $\Gamma$ ) govern the scaled manyparticle probability distribution.

It is useful to express these parameters in terms $\phi$, which leads to

$$
\tilde{\lambda}=\frac{\delta^{3}}{\sigma^{2} \gamma} \phi \quad \text { and } \quad \Gamma=\frac{u_{0}}{k_{\mathrm{B}} T} \frac{n \delta}{\gamma} \phi^{n+1} .
$$

The generalization to higher dimensions is straightforward. For example, in 2D, where $\phi=N \gamma^{2} / V$ and $d=\sqrt{V /(N \pi)}=$ $\gamma / \sqrt{\pi \phi}$, the two relevant parameters are

$$
\tilde{\lambda}=\frac{\delta^{3}}{\sigma^{2} \gamma} \sqrt{\pi \phi} \quad \text { and } \quad \Gamma=\frac{u_{0}}{k_{\mathrm{B}} T} \frac{n \delta}{\gamma}(\pi \phi)^{(n+1) / 2} .
$$

In this form, the dimensionality primarily enters into the $\phi$ dependence of these two parameters (see eq. (32)). The validity of these reduced parameter sets is confirmed by numerical simulations (see Fig. 10.

As discussed earlier in the single-particle case, this parameter reduction does not extend to the passive regime. In this case a diffusive scaling is required

$$
\tilde{t}=\frac{t}{d^{2}}, \quad \tilde{x}(\tilde{t})=\frac{x(t)}{d},
$$

in terms of which the Fokker-Planck equation becomes 24

$$
\frac{\partial}{\partial \tilde{t}} \tilde{P}_{\tilde{t}}[\tilde{\mathbf{x}}]=-\frac{1}{6} \Gamma_{0} \sum_{i} \frac{\partial}{\partial \tilde{x}_{i}}\left\{\sum_{j \neq i} \frac{1}{\left(\tilde{x}_{i}-\tilde{x}_{j}\right)^{7}} P_{\tilde{t}}[\tilde{\mathbf{x}}]\right\}+\frac{1}{6} \sum_{i} \frac{\partial^{2}}{\partial \tilde{x}_{i}^{2}} P_{\tilde{t}}[\tilde{\mathbf{x}}]
$$

with

$$
\Gamma_{0}=\left\{\begin{array}{l}
\frac{n u_{0}}{k_{\mathrm{B}} T} \phi^{n}, \text { in } 1 \mathrm{D} \\
\frac{n u_{0}}{k_{\mathrm{B}} T}(\pi \phi)^{n / 2}, \text { in } 2 \mathrm{D}
\end{array}\right.
$$

Therefore, the probability only depends $\frac{14}{14}$ on $\Gamma_{0}$, which differs from $\Gamma$.

In summary, the small- $\delta$ limit of the systems described in this work is described by two relevant parameters for the active regime and a single relevant parameter for the passive regime. There is no smooth transition from one regime to the other when $\delta$ is small. This is because the limits $\delta \rightarrow 0$ and 

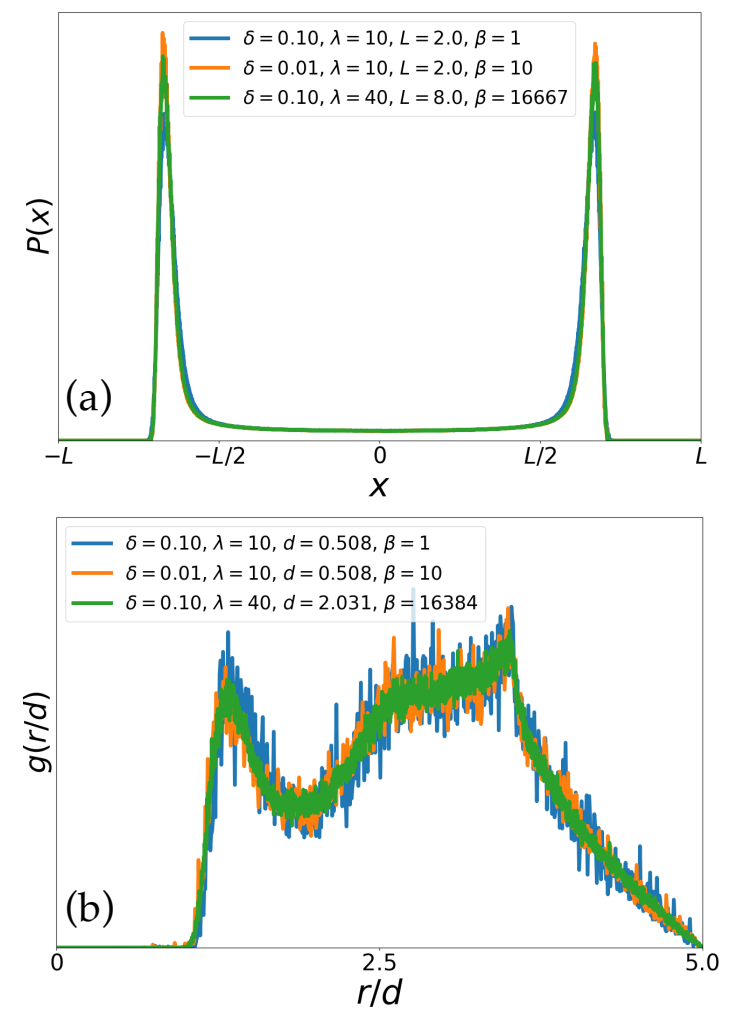

FIG. 10. Numerical verification of the reduced set of relevant parameters in the active limit (finite $\lambda$ ). (a): 1D single-particle case. Histogram of the particle position in a confining potential $U(x)=$ $u_{0} \gamma^{6}\left((L+x)^{-6}+(L-x)^{-6}\right)$. Data for different $\delta, \lambda$ and $\beta$, but constant $\lambda / L=2.5$ and $\beta u_{0} \delta \gamma^{6} / L^{7}=6.209 \times 10^{-6}$. (b): Twodimensional many-particle case. Pair-correlation function $g(r)$ for $N=16$ and $n=6$. Data for different $\delta, \lambda$ and $\beta$, at constant $\lambda / d=11.1$ and $\beta u_{0} \delta \gamma^{6} / d^{7}=0.209$. (All data for $\gamma=1, u_{0}=1$.)

$\lambda \rightarrow 0$ are not exchangeable. Therefore, a phase diagram covering both passive and active is not possible on a two parameter space. A complete phase diagram is three dimensional on a rescaled parameter space $\delta / d, \lambda / d$ and $\Gamma_{0}$ in eq. (36). Expressed in terms of $\phi$, they yield

$$
\begin{aligned}
& \frac{\delta}{\gamma} \phi, \frac{\lambda}{\gamma} \phi, \text { and } \Gamma_{0}, \quad \text { for } 1 \mathrm{D} \\
& \frac{\delta}{\gamma} \sqrt{\pi \phi}, \frac{\lambda}{\gamma} \sqrt{\pi \phi} \text {, and } \Gamma_{0}, \text { for } 2 \mathrm{D} \text {. }
\end{aligned}
$$

\section{Continuous-time description}

In the analysis presented so far, the Fokker-Planck description was obtained in the small- $\delta$ limit. However, it is possible 24 to define a continuous-time description of the MC dynamics for arbitrary values of $\delta$. This can be obtained using the Kramers-Moyal expansion ${ }^{26}$ of the Master equation eq. (17), which leads to the Fokker-Planck equation

$$
\begin{aligned}
\partial_{t} P_{t}(x, \varepsilon)= & \frac{\sigma^{2}}{2} \frac{\partial^{2}}{\partial \varepsilon^{2}} P_{t}(x, \varepsilon)+f(x-\varepsilon, \varepsilon) P_{t}(x-\varepsilon, \varepsilon) \\
& -f(x, \varepsilon) P_{t}(x, \varepsilon)
\end{aligned}
$$

with the reflecting boundary at $\varepsilon= \pm \delta$ as zero-current condition

$$
\left.\frac{\partial P_{t}(x, \varepsilon)}{\partial \varepsilon}\right|_{\varepsilon= \pm \delta}=0
$$

Taking the small- $\delta$ limit of eq. (37), we recover the scaling of eq. (33) that was obtained from the discrete-time Master equation eq. (17).

\section{Active random acceleration process}

An intriguing aspect of the dimensional reduction of the phase space is the singular nature of the passive case on a two-parameter plane for $\delta \rightarrow 0$. This singular behavior of the rescaled parameters is not due to the discrete nature of the MC dynamics as it also appears in the continuous-time description discussed above. For the kinetic MC, the singularity appears in the small- $\delta$ limit. However, it can also appear in other stochastic models of active matter, even without taking a vanishing limit of a parameter similar to $\delta$. To illustrate this point, we define a continuous model which closely resembles the kinetic MC dynamics. This model, which we refer as the active random acceleration process ${ }^{27}$ is defined for continuous time by a coupled Langevin equation

$$
\begin{aligned}
& \dot{\varepsilon}_{t}=r_{t}+R\left(\frac{\varepsilon_{t}}{\delta}\right), \\
& \dot{x}_{t}=v_{0} \varepsilon_{t}+\frac{1}{k_{\mathrm{B}} T} F\left(x_{t}\right)+s_{t},
\end{aligned}
$$

where $x_{t}$ is the position of the self-propelled particle at time $t$. The two noise terms, $r_{t}$ and $s_{t}$, are Gaussian white noises with zero mean and covariance $\left\langle r_{t} r_{t^{\prime}}\right\rangle=\sigma^{2} \delta\left(t-t^{\prime}\right)$ and $\left\langle s_{t} s_{t^{\prime}}\right\rangle=$ $2 D \delta\left(t-t^{\prime}\right)$, respectively. The term $R$ denotes the reflecting boundary condition at $\pm \delta$. The parameter $v_{0}$ characterizes the strength of the self-propulsion 28 .

Similar to the kinetic MC dynamics, in the scaled coordinates $t \rightarrow t d / \delta, \varepsilon \rightarrow v \delta, x \rightarrow x d$ the dynamics eq. (39) is described by the Fokker-Planck equation

$$
\begin{aligned}
& \frac{\partial}{\partial t} P_{t}(x, v)=\frac{d}{2 \lambda} \frac{\partial^{2}}{\partial v^{2}} P_{t}(x, v)+\frac{D}{\delta d} \frac{\partial^{2}}{\partial x^{2}} P_{t}(x, v) \\
& -\frac{1}{\delta k_{\mathrm{B}} T} \frac{\partial}{\partial x}\left[F(x d) P_{t}(x, v)\right]-v_{0} \frac{\partial}{\partial x}\left[v P_{t}(x, v)\right]
\end{aligned}
$$

Its multi-particle generalization is straightforward. For the inter-particle force eq. 26, the steady-state probability distribution is controlled by three parameters

$$
A=\frac{D}{\delta d} \frac{\lambda}{d}, B=v_{0} \frac{\lambda}{d}, \text { and } C=\frac{n u_{0} \gamma^{n}}{k_{\mathrm{B}} T \delta d^{n+1}} \frac{\lambda}{d} .
$$


The passive limit corresponds to $v_{0}=0$, where the $x$ and $v$ coordinates decouple and the probability $P(x, v)=P(x) P(v)$. In such case, we see that the $P(x)$ follows the usual equilibrium Fokker-Planck equation and the steady-state $P(x)$ is determined by a single control parameter $C / A$, which coincides with $\Gamma_{0}$ for $D=1$. Then, importantly, $P(x) \propto \exp (-C u(x) / A)$, where $u(x)$ is the rescaled potential and therefore, setting $D=0$ makes the probability distribution uniform and independent of the inter-particle interaction, which corresponds to an infinite temperature.

In the active limit, there are the three control parameters $A$, $B$, and $C$. The only way to reduce the number of parameters, while keeping the particles active and interacting, is by setting $D=0$ and thereby $A=0$. However, this would make the finitetemperature passive limit inaccessible, as we discussed above. This shows that the singular behavior of the MC dynamics, when trying to reduce the number of control parameters to two also happens in this active random acceleration process. A similar singular behavior is present for other models. We have checked ${ }^{24}$ that the same statement applies for the active Ornstein-Uhlenbeck process.

From this analysis, we can conclude that in these classes of active dynamics, at least three independent relevant parameters are required to describe the full phase diagram including the passive regime.

\section{CONCLUSIONS}

In this work, we presented a kinetic-Monte Carlo perspective on two-dimensional active matter. Within this approach, we established (in extension of our earlier work ${ }^{8}$ ) the presence of the liquid, hexatic, and solid active-matter phases from their constituent decay laws of positional and orientational order (see Table I). We also ascertained continuity of the active-matter phases in the passive limit and recovered the phases of the corresponding equilibrium system. We have not tested very soft repulsive inverse-power-law potentials $(n<6$ in eq. (4), but expect on the basis of our findings that for sufficiently steep repulsive inverse-power-law potentials the twostep melting behavior of the equilibrium system is maintained up to high activities, and possibly up to infinite persistence lengths. The stability of the intermediate hexatic phase in the high-activity regime - far above the linear-response regimeis intriguing. It may be due to an underlying symmetry that is yet to be understood. We have not addressed here the nature of the melting transitions in the active region, that is, the question of whether the liquid-hexatic phase transition is of first order or of Kosterlitz-Thouless type e $^{29[30}$ (as in the equilibrium system ${ }^{14}$ ), and whether this theory continues to apply at all to the hexatic-solid transition in active systems.

Besides the melting phase transitions, we have established the existence of the MIPS phase and identified it as a coexistence between a liquid and a gas: two phases with exponential decays of both correlation functions, but with typically very different densities. The coexistence region was identified for a wide range of interaction potentials including the hard-disk limit. It remains disjoint from the melting lines for all values of $n$ (possibly excluding very soft potentials). An analogy of the MIPS with the liquid-gas coexistence in equilibrium indicates the existence ${ }^{31}$ of a critical point, although we have not studied it in detail.

We also discussed the dimensionality of the phase diagram. We showed that the qualitative phase behavior is robust against changes of the maximum step size $\delta$. The steady state reached by the kinetic MC dynamics is fully described by the density, the persistence length, and the maximum step size $\delta$, although a two-dimensional scaling describes the MIPS phase and the melting transitions at high density $\phi$ for small $\delta$. We argued in this direction using the stochastic description of the kinetic MC dynamics, and its formulation in terms of a Langevin dynamics. The common scaling with $\delta$ of the melting transitions and of the MIPS breaks down in the vicinity of the equilibrium phase transition point. This is needed to obtain the one-parameter scaling (for an inverse-power-law potential) in the passive limit.

The detailed phase diagrams found in our present work once more illustrate the rich collective properties in nonequilibrium physics, and the current limits of our understanding of these models and, more generally, of the physics of active matter.

\section{ACKNOWLEDGMENTS}

We thank H. Löwen and L. Berthier for helpful discussions. W.K. acknowledges support from the Alexander von Humboldt Foundation.

\section{Appendix A: Passive Fokker-Planck equation under diffusive scaling}

In the passive limit, it follows from (15) that $g\left(\varepsilon, \varepsilon^{\prime}\right)=$ $1 /(2 \delta)$. In this case, the Master equation (of eq. [17) can be recast by using the definition $P_{k}(x)=\int_{-\delta}^{\delta} d \varepsilon P_{k}(x, \varepsilon)$ and integrating over $\varepsilon^{\prime}$ leads to

$$
\begin{aligned}
& P_{k+1}(x)=P_{k}(x)+\frac{1}{2 \delta} \int_{-\delta}^{\delta} d \varepsilon \\
& \left\{f(x-\varepsilon, \varepsilon) P_{k}(x-\varepsilon)-f(x, \varepsilon) P_{k}(x)\right\} .
\end{aligned}
$$

Applying the diffusive scaling in eq. 21, it follows

$$
\begin{aligned}
& \tilde{P}_{t+\delta^{2}}(x)=\tilde{P}_{t}(x)+\frac{1}{2} \int_{-1}^{1} d v \\
& \left\{f(x-\delta v, \delta v) \tilde{P}_{t}(x-\delta v)-f(x, \delta v) \tilde{P}_{t}(x)\right\},
\end{aligned}
$$

with $\tilde{P}_{t}(x)=P_{k}(x)$. Expanding in powers of small $\delta$ leads to

$\tilde{P}_{t+\delta^{2}}(x)=\tilde{P}_{t}(x)-\frac{\delta}{2} \frac{d}{d x}\left[A(x) \tilde{P}_{t}(x)\right]+\frac{\delta^{2}}{4} \frac{d^{2}}{d x^{2}}\left[B(x) \tilde{P}_{t}(x)\right]+\cdots$,

with the definitions

$$
A(x)=\int_{-1}^{1} d v v f(x, \delta v), \text { and } B(x)=\int_{-1}^{1} d v v^{2} f(x, \delta v) .
$$


The expression for $f(x, \varepsilon)$ in eq. 6b can be rewritten as

$$
\begin{array}{r}
f(y, \varepsilon)=1-\Theta\left(\frac{U(y+\varepsilon)-U(y)}{k_{\mathrm{B}} T}\right) \\
\left\{1-\exp \left(-\frac{U(y+\varepsilon)-U(y)}{k_{\mathrm{B}} T}\right)\right\},
\end{array}
$$

with $\Theta(x)$ the Heaviside function. Using this expression in $A(x)$ in eq. A3 and expanding in terms of small $\delta$, it follows

$$
A(x)=\beta \delta F(x) \int_{-1}^{1} d v v^{2} \Theta(-v F(x))+\cdots
$$

with the force $F(x)=-\frac{\partial}{\partial x} U(x)$. The two cases $F(x)>0$ and $F(x)<0$ lead to the same result for the integral (namely $1 / 3$ ), thus giving

$$
A(x)=\beta \frac{\delta F(x)}{3}+\cdots
$$

In the same way it follows for $B(x)$ in eq. A3 (the term with $\Theta(.$.$) contributes to order \delta$ and is thus neglected)

$$
B(x)=\frac{2}{3}+\mathscr{O}(\delta)
$$

Using this expressions of eqs $\mathrm{A} 4$ and $\mathrm{A} 5$ in eq. $\mathrm{A} 2 \mathrm{re}$ sults in the well-known Fokker-Planck equation for a passive particle in a potential

$$
\frac{\partial \tilde{P}_{t}(x)}{\partial t}=-\frac{1}{k_{\mathrm{B}} T} \frac{\partial}{\partial x}\left[F(x) \tilde{P}_{t}(x)\right]+\frac{\partial^{2}}{\partial x^{2}}\left[\tilde{P}_{t}(x)\right] .
$$

This corresponds to the standard Langevin description of a passive particle

$$
\dot{x}(t)=\beta F(x)+\eta(t) \quad\left\langle\eta(t) \eta\left(t^{\prime}\right)\right\rangle=2 \delta\left(t-t^{\prime}\right) .
$$

The steady-state probability $P(x) \sim \exp (-\beta U(x))$, thus the single relevant parameter is $\beta U(x)$.

If the ballistic scaling of eq. (8) was used for the passive case, it would result in

$$
\frac{\partial P_{t}(x)}{\partial t}=\frac{\Gamma_{1}}{6} \frac{\partial}{\partial x}\left[F(x) P_{t}(x)\right]
$$

which clearly does not capture the correct physics.

\footnotetext{
${ }^{1}$ M. E. Cates, "Diffusive transport without detailed balance in motile bacteria: does microbiology need statistical physics?" Rep. Prog. Phys 75, 042601 (2012).

${ }^{2}$ G. S. Redner, M. F. Hagan, and A. Baskaran, "Structure and Dynamics of a Phase-Separating Active Colloidal Fluid," Phys. Rev. Lett. 110, 055701 (2013).

${ }^{3}$ Y. Fily and M. C. Marchetti, "Athermal Phase Separation of Self-Propelled Particles with No Alignment," Phys. Rev. Lett. 108, 235702 (2012).

${ }^{4}$ T. Speck, J. Bialké, A. M. Menzel, and H. Löwen, "Effective Cahn-Hilliard Equation for the Phase Separation of Active Brownian Particles," Phys. Rev. Lett. 112, 218304 (2014).
}

${ }^{5}$ J. Bialké, T. Speck, and H. Löwen, "Crystallization in a Dense Suspension of Self-Propelled Particles,” Phys. Rev. Lett. 108, 168301 (2012).

${ }^{6}$ L. F. Cugliandolo, P. Digregorio, G. Gonnella, and A. Suma, "Phase coexistence in bidimensional passive and active dumbbell systems," Phys. Rev. Lett. 119, 268002 (2017).

${ }^{7}$ D. Levis and L. Berthier, "Clustering and heterogeneous dynamics in a kinetic Monte Carlo model of self-propelled hard disks," Phys. Rev. E 89, 062301 (2014).

${ }^{8}$ J. U. Klamser, S. C. Kapfer, and W. Krauth, "Thermodynamic phases in two-dimensional active matter," Nat. Commun. 9, 5045 (2018).

${ }^{9}$ N. D. Mermin, "Crystalline order in two dimensions," Phys. Rev. 176, 250 254 (1968).

${ }^{10}$ B. I. Halperin and D. R. Nelson, "Theory of Two-Dimensional Melting," Phys. Rev. Lett. 41, 121-124 (1978).

${ }^{11}$ D. R. Nelson and B. I. Halperin, "Dislocation-mediated melting in two dimensions," Phys. Rev. B 19, 2457-2484 (1979).

${ }^{12}$ A. P. Young, "Melting and the vector Coulomb gas in two dimensions," Phys. Rev. B 19, 1855-1866 (1979).

${ }^{13}$ E. P. Bernard and W. Krauth, "Two-Step Melting in Two Dimensions: FirstOrder Liquid-Hexatic Transition,” Phys. Rev. Lett. 107, 155704 (2011).

${ }^{14}$ S. C. Kapfer and W. Krauth, "Two-Dimensional Melting: From LiquidHexatic Coexistence to Continuous Transitions," Phys. Rev. Lett. 114, 035702 (2015).

${ }^{15}$ P. Romanczuk, M. Bär, W. Ebeling, B. Lindner, and L. SchimanskyGeier, "Active Brownian particles," Eur. Phys. J. Special Topics 202, 1-162 (2012).

${ }^{16}$ É. Fodor, C. Nardini, M. E. Cates, J. Tailleur, P. Visco, and F. van Wijland, “How Far from Equilibrium Is Active Matter?” Phys. Rev. Lett 117, 038103 (2016).

${ }^{17}$ W. Krauth, Statistical Mechanics: Algorithms and Computations (Oxford University Press, 2006).

${ }^{18}$ N. D. Mermin and H. Wagner, "Absence of Ferromagnetism or Antiferromagnetism in One- or Two-Dimensional Isotropic Heisenberg Models," Phys. Rev. Lett. 17, 1133-1136 (1966).

${ }^{19}$ N. D. Mermin, "Crystalline Order in Two Dimensions," Physical Review 176, 250-254 (1968)

${ }^{20}$ W. Mickel, S. C. Kapfer, G. E. Schröder-Turk, and K. Mecke, "Shortcomings of the bond orientational order parameters for the analysis of disordered particulate matter," J. Chem. Phys. 138, 044501 (2013).

${ }^{21}$ I. Buttinoni, J. Bialké, F. Kümmel, H. Löwen, C. Bechinger, and T. Speck, "Dynamical Clustering and Phase Separation in Suspensions of SelfPropelled Colloidal Particles," Phys. Rev. Lett. 110, 238301 (2013).

${ }^{22}$ P. Digregorio, D. Levis, A. Suma, L. F. Cugliandolo, G. Gonnella, and I. Pagonabarraga, "Full Phase Diagram of Active Brownian Disks: From Melting to Motility-Induced Phase Separation,” Phys. Rev. Lett. 121, 098003 (2018).

${ }^{23}$ The persistence length used for the numerical results eq. (1) differs by a numerical constant in both one and two dimensions.

${ }^{24} \mathrm{~J}$. U. Klamser, Low-dimensional phase transitions in and outside equilibrium, Ph.D. thesis, SORBONNE UNIVERSITÉ (2018).

${ }^{25}$ The approximate dynamics in eq. (6) has a different Master equation. Nevertheless, both describe the same dynamics in the small- $\delta$ limit.

${ }^{26}$ N. V. Kampen, Stochastic Processes in Physics and Chemistry, Vol. 3 (Elsevier, 2007).

${ }^{27}$ S. N. Majumdar, A. Rosso, and A. Zoia, "Time at which the maximum of a random acceleration process is reached," J. Phys. A: Math. Theor. 43, 115001 (2010).

${ }^{28}$ Putting $1 / k_{\mathrm{B}} T$ in the force term of eq. $39 \mathrm{~b}$ is motivated by the aim to compare with the kinetic $\mathrm{MC}$ results.

${ }^{29}$ J. M. Kosterlitz and D. J. Thouless, "Long range order and metastability in two dimensional solids and superfluids. (Application of dislocation theory)," J. Phys. Condens. Matter 5, L124-L126 (1972).

${ }^{30}$ J. M. Kosterlitz and D. J. Thouless, "Ordering, metastability and phase transitions in two-dimensional systems," J. Phys. C 6, 1181-1203 (1973).

${ }^{31}$ J. T. Siebert, F. Dittrich, F. Schmid, K. Binder, T. Speck, and P. Virnau, "Critical behavior of active Brownian particles," Phys. Rev. E 98 (2018). 